Pecvnia, núm. 12 (enero-junio, 2011), pp. 119-132

\title{
LA EMPRESA FAMILIAR EN LA LEY DE SDCIEDADES DE CAPITAL: IMPLICACIONES FISCALES
}

fecha de recepción: 03/03/2011

fecha de aceptación: 12/04/2011

\author{
Alfonso García-Moncó ${ }^{1}$ \\ a.monco@uah.es
}

Universidad de Alcalá de Henares

\section{Resumen}

La Sociedad Limitada Nueva Empresa puede ser el vehículo idóneo para la adopción de la forma societaria por una empresa familiar. El Real Decreto Legislativo 1/2010, de 3 de julio que aprueba el Texto Refundido de la Ley de Sociedades de Capital incorpora en su articulado y en sus disposiciones adicionales importantes ventajas para las SLNE, tanto para facilitar su constitución, como para flexibilizar su funcionamiento y reducir los costes fiscales de su creación. Las empresas familiares de reducida dimensión pueden beneficiarse de este marco jurídico y fiscal que, en estos momentos, no tiene comparación con ninguna otra modalidad societaria. Sin embargo, esta realidad es, en gran parte, desconocida porque no se han explicado las posibilidades que contiene la Ley de Sociedades de Capital al respecto y sus consecuencias fiscales.

Palabras clave: Empresa familiar; Sociedad Limitada Nueva Empresa; Ley de Sociedades de Capital; Constitución de Sociedades; Beneficios fiscales.

\section{Abstract}

The New Enterprise Limited Company can be an ideal vehicle for the adoption of the corporate form by a family business. The Royal Decree $1 / 2010$ of 3 July, which approves the revised text of the Capital Company Act, includes in its provisions and its additional provisions for SLNE important advantages, both to facilitate their establishment, as to ease operation and reduce the fiscal costs of its creation. The small size family businesses can benefit from the legal and fiscal framework at the moment, is unmatched by any other corporate form.

1 Catedrático de Derecho Financiero y Tributario. Facultad de Derecho de la Universidad de Alcalá, C/ Libreros, 27, E-28801, Alcalá de Henares, Madrid. 
However, this reality is largely unknown because they have not explained the possibilities contained in the Companies Act and Capital about the tax consequences.

Keywords: Family business/Family enterprise; New enterprise limited company; Capital companies act; Company formation; Tax benefits.

\section{Introducción}

El Real Decreto Legislativo 1/2010, de 3 de julio que aprueba el Texto Refundido de la Ley de Sociedades de Capital constituye sin duda un hito en la evolución no sólo del Derecho Societario sino de todo el Ordenamiento Jurídico. El origen de este cuerpo normativo, como señala la Exposición de Motivos del Real Decreto-Legislativo, es el siguiente: "El presente real decreto legislativo cumple con la previsión recogida en la disposición final séptima de la Ley 3/2009, de 3 de abril, sobre modificaciones estructurales de las sociedades mercantiles, que habilita al Gobierno para que, en el plazo de doce meses, proceda a refundir en un único texto, bajo el título de «Ley de Sociedades de Capital», las normas legales que esa disposición enumera. De este modo se supera la tradicional regulación separada de las formas o tipos sociales designadas con esa genérica expresión, que ahora, al ascender a título de la ley, alcanza rango definidor".

La regulación que prevea un Estado de Derecho del marco jurídico de las sociedades resulta decisiva en el orden jurídico, económico y empresarial. Pues bien, en la realidad empresarial se reconoce cada vez más importancia a las llamadas empresas familiares constituidas precisamente en torno a un núcleo familiar. Este origen no debe sin embargo sugerir que nos movemos únicamente en el ámbito de la pequeña y mediana empresa. En España existen grandes sociedades de capital que son empresas familiares. Po otra parte, el carácter familiar de una empresa no prejuzga el ropaje societario que pueda adoptar y que, en principio, puede afectar a cualquiera de las figuras previstas en la Ley. No obstante prestaremos especial atención a alguna de las formas societarias que se adaptan singularmente bien a este sector como es la Sociedad Limitada Nueva Empresa.

Dichas empresas con su peculiar problemática tienen su proyección en la Ley de Sociedades de Capital y este va a ser el objeto del presente trabajo, examinar su regulación para después analizar sus implicaciones fiscales.

\section{Concepto de empresa familiar}

Aunque existen distintas aproximaciones a la realidad mencionada a nosotros nos interesa sobre todo la perspectiva fiscal y desde la misma se considera empresa o en su caso sociedad familiar, según el artículo 4.8 apartado dos, letra b) de la Ley del Impuesto de Patrimonio, el siguiente el ámbito subjetivo, establecido para delimitar la exención: 
"Que la participación del sujeto pasivo en el capital de la entidad sea al menos del 5 $\%$ computado de forma individual, o del $20 \%$ conjuntamente con su cónyuge, ascendientes, descendientes o colaterales de segundo grado, ya tenga su origen el parentesco en la consanguinidad, en la afinidad o en la adopción".

Es decir, una empresa familiar es la formada por el sujeto pasivo, el cónyuge, ascendientes, descendientes o colaterales de segundo grado por consanguinidad, afinidad o adopción. En dichos límites, se considera aplicable la normativa fiscal que se refiera a la llamada "empresa familiar" y por tanto también a la sociedad familiar cuando la empresa adquiera forma societaria.

Como se puede observar no hay ningún límite cuantitativo en lo que se refiere al valor de las acciones o participaciones de las sociedades de capital ni a su número ni a su valor nominal. Por tanto, respecto a las consecuencias fiscales de la referida calificación, ésta no depende de los últimos factores aludidos sino única y exclusivamente de la existencia del citado núcleo familiar y de la participación mínima prevista por el artículo trascrito de la Ley del Impuesto de Patrimonio. Es más, el Real Decreto 1704/1999, de 5 de noviembre, por el que se determinan los requisitos y condiciones de las actividades empresariales y profesionales y de las participaciones en entidades para la aplicación de las exenciones correspondientes en el Impuesto sobre el Patrimonio (BOE núm. 266 de 6 de noviembre) precisa en su artículo 5 lo siguiente:

"Cuando la entidad participe a su vez en otras entidades, se considerará que no realiza una actividad de gestión de un patrimonio mobiliario, si disponiendo directamente al menos del 5 por 100 de los derechos de voto en dichas entidades, dirige y gestiona el conjunto de las actividades económicas de éstas mediante la correspondiente organización de medios personales y materiales, siempre que las entidades participadas no tengan a su vez como actividad principal la gestión de un patrimonio mobiliario o inmobiliario o concurra, en las mismas, alguno de los supuestos previstos en los párrafos a) y c) del apartado 1 del artículo 75 de la Ley del Impuesto sobre Sociedades".

Es decir, el reglamento que interpreta la exención prevista en la Ley del Impuesto sobre el Patrimonio que -por otra parte debemos recordar que sigue plenamente en vigor aunque la Ley 4/2008, haya derogado los preceptos relativos a la obligación de declarar y pagaradmite la existencia de lo que podríamos denominar "holding familiar" que se puede reconocer fiscalmente con la participación de un 5\% de los derechos de voto en otras entidades siempre que sea realmente un holding con medios personales y materiales y no una simple sociedad de "cartera". Aunque esto se diga a efectos de evitar que el favorable tratamiento tributario que se reconoce a las participaciones en dichas entidades se adjudique a simples sociedades de inversión, lo cierto es que supone admitir que las sociedades familiares no tienen, como decimos más límite que la expresada participación mínima del $5 \%$ en los derechos de voto y no restricciones de otro tipo ni siquiera su eventual cotización en Bolsa. 


\section{La Sociedad Limitada Nueva Empresa como vehículo jurídico de la empresa familiar}

Ante todo hay que aclarar que el hecho de que nos refiramos, de forma particular, a esta figura societaria no quiere decir -ya lo hemos adelantado- que no haya sociedades familiares bajo el modelo jurídico de otras formas de entidades como las sociedades limitadas del régimen general o en las sociedades anónimas. Muy al contrario, en España existen no sólo sociedades sino importantes grupos societarios que son núcleos familiares como es el caso de las sociedades agrupadas en el Instituto de Empresa Familiar. Como acabamos de destacar es perfectamente posible que un gran grupo societario que cotice en Bolsa se una empresa familiar a efectos fiscales.

No obstante, nos vamos a ocupar prioritariamente de la Sociedad Limitada Nueva Empresa porque creemos que ofrece posibilidades muy interesantes sobre todo para las empresas familiares que inician su actividad y por ello, las tratamos de forma singularizada, sin perjuicio de que hagamos cuantas referencias sean necesarias a las demás fórmulas societarias.

Los artículos 434 a 454 de la Ley de Sociedades de Capital configuran un marco jurídico especialmente adecuado para el inicio de la actividad de una empresa familiar. Las facilidades de constitución que concede El Texto Refundido de la Ley que veremos seguidamente así como la simplicidad de su régimen jurídico y las ventajas fiscales que describiremos en el epígrafe siguiente, favorecen su adaptación a las necesidades de una empresa de dichas características.

Así podemos mencionar los siguientes aspectos:

- Carácter personalísimo de la Sociedad, vinculado a un socio que da sus apellidos a la sociedad, (artículo 435.1) con un número máximo de cinco socios, siempre personas físicas (artículo 437) y que puede ser también sociedad unipersonal (artículo 438). Igualmente refuerzan dicho carácter personalísimo los requisitos subjetivos para la transmisión de las participaciones personales (artículo 444) que deberán transmitirse siempre a personas físicas.

- El órgano de administración de la Sociedad se ajusta especialmente bien a la realidad a la que nos referimos porque sólo contempla la figura del Administrador y excluye el Consejo de Administración (artículo 447).

- Igualmente su reducido capital social con un mínimo de 3.012 € favorece a las pequeñas empresas, aunque consideramos criticable que el máximo sea de $120.202 €$, demasiado reducido para nuestros días y asimismo creemos que es una limitación innecesaria que deba ser desembolsado exclusivamente en dinero y no se permita que una parte del mismo pueda consistir en bienes de otra naturaleza como por ejemplo un local de negocio.

- La configuración del objeto social (artículo 436) también se adapta muy bien a las exigencias que puede plantear una sociedad familiar al mencionar muchos de los sectores de actividades económicas donde habitualmente se sitúan esta clase de empresas como la agrícola, ganadera, pesquera, turística o las actividades profesionales y de intermediación. No obstante, también se aceptan "cualquier 
actividad singular distinta de las anteriores" (artículo 436.2). En este sentido, hay que mencionar especialmente la actividad de compraventa y arrendamiento de bienes inmuebles que, por las exigencias de la normativa fiscal, a las que nos referimos más adelante, puede cumplir perfectamente una sociedad familiar.

- El régimen establecido para la retribución del administrador, de carácter potestativo, "podrá ser retribuido" y flexible, "en la forma y cuantía que decida la junta general" (artículo 448.2) se acopla perfectamente a las necesidades de una sociedad familiar y, como veremos, a las exigencias de la Ley del Impuesto de Patrimonio para aplicar la exención a las participaciones de dichas sociedades.

- La regulación prevista en la Ley para el usufructo de participaciones se acomoda especialmente bien a las sociedades familiares que se constituyan como SLNE dado que, como es lógico, este derecho es muy importante, teniendo en cuenta la habitual transmisión mortis causa de las participaciones en la que juega un papel esencial, el usufructo del cónyuge supérstite.

- Además de las facilidades establecidas en lo que se refiere a los requisitos constitutivos de la SLNE en los artículos 439 a 442 de la Ley, las disposiciones adicionales de la misma, $3^{a}, 4^{a}$ y $6^{a}$ establecen un cuadro normativo, particularmente en materia tributaria, notablemente beneficioso para las sociedades que tengan esta calificación.

\section{Implicaciones fiscales en la nueva Ley para las Sociedades Familiares y en particular para las SLNE}

El marco jurídico-fiscal de las SLNE viene constituido por una serie de elementos que vamos a enunciar siguiendo los aspectos más relevantes de su regulación en la Ley de Sociedades de Capital:

Primero. Con carácter previo, no debe olvidarse lo dispuesto respecto de las denominadas "cuentas-ahorro nueva empresa" en el artículo 68.6 de la Ley 35/2006, de 28 de noviembre del Impuesto sobre la Renta de las Personas Físicas (en adelante IRPF) y de modificación parcial de las Leyes de los Impuestos sobre Sociedades, sobre la Renta de no Residentes y sobre el Patrimonio (BOE núm. 285 de 29 de noviembre) que dispone en su primer párrafo:

"Los contribuyentes podrán aplicar una deducción por las cantidades que depositen en entidades de crédito, en cuentas separadas de cualquier otro tipo de imposición, destinadas a la constitución de una Sociedad Nueva Empresa regulada en el capítulo XII de la ley 2/1995 de 23 de Marzo, de Sociedades de Responsabilidad Limitada...”.

Es decir, la constitución de una SLNE puede planificarse, iniciando las actuaciones con la apertura de una o varias cuentas de ahorro nueva empresa donde, por ejemplo, se puede depositar el capital que se va a desembolsar posteriormente, obteniendo así desde el primer momento, una rentabilidad fiscal de las aportaciones.

Segundo. Uno de los primeros aspectos en que el Texto Refundido de la Ley de Sociedades de Capital, beneficioso para las empresas familiares, es el que se refiere al régimen jurídico del usufructo de participaciones (artículo 127), muy relevante en las 
mismas, dado que en la estructura habitual de la sucesión "mortis causa", el usufructo del cónyuge supérstite está siempre presente. A tal efecto se establece en su apartado primero que "en caso de usufructo de participaciones o de acciones la cualidad de socio reside en el nudo propietario, pero el usufructuario tendrá derecho en todo caso a los dividendos acordados por la sociedad durante el usufructo. Salvo disposición contraria de los estatutos, el ejercicio de los demás derechos del socio corresponde al nudo propietario."

Conviene recordar que uno de los aspectos esenciales del régimen tributario privilegiado de que disfrutan las sociedades familiares es la exención de sus participaciones en el Impuesto de Sucesiones y Donaciones. Los requisitos establecidos en el artículo 20.2.c) de la Ley 29/1987 de 18 de Diciembre reguladora del Impuesto de Sucesiones y Donaciones para aplicar una reducción del 95\% son los siguientes:

"En los casos en los que en la base imponible de una de una adquisición "mortis causa" que corresponda a los cónyuges, descendientes o adoptados de la persona fallecida, estuviese incluido el valor de una empresa individual, de un negocio profesional o participaciones en entidades, a los que sea de aplicación la exención regulada en el apartado octavo, del artículo 4 de la Ley 19/1991, de 6 de Junio, del Impuesto sobre el Patrimonio, o el valor de los derechos de usufructo sobre los mismos, o de derechos económicos derivados de la extinción de dicho usufructo, siempre que con motivo del fallecimiento se consolidara el pleno dominio en el cónyuge descendientes o adoptados, o percibieran éstos los derechos debidos a la finalización del usufructo en forma de participaciones en la empresa negocio o entidad afectada, para obtener la base liquidable se aplicará en la imponible, con independencia de las reducciones que procedan de acuerdo con los apartados anteriores, otra del 95 por 100 del mencionado valor, siempre que la adquisición se mantenga, durante los diez años siguientes al fallecimiento del causante, salvo que falleciera el adquirente dentro de ese plazo”.

Debe aclararse al respecto de la alusión del precepto trascrito al usufructo y a la consolidación del dominio que el artículo $4^{\circ}$ la Ley 62/2003 de 30 de diciembre de Medidas Fiscales Administrativas y de Orden Social para 2004 modificó la Ley del Impuesto de Patrimonio en este punto, admitiendo que la reducción se produzca tanto en la situaciones de plena propiedad, la nuda propiedad y el derecho de usufructo vitalicio sobre las participaciones en entidades.

En cuanto a una eventual transmisión lucrativa "inter vivos" a través de una donación, que como hemos visto, es posible en virtud del artículo 444.2 siempre que se haga a favor de personas físicas, se aplica el mismo régimen descrito según lo dispuesto en el artículo 20.6 de la misma Ley de Sucesiones siempre que se cumplan los siguientes requisitos:

“a) Que el donante tuviese sesenta y cinco o más años o se encontrase en situación de incapacidad permanente, en grado de absoluta o gran invalidez.

b) Que, si el donante viniere ejerciendo funciones de dirección, dejara de ejercer y de percibir remuneraciones o por el ejercicio de dichas funciones desde el momento de la transmisión. 
A estos efectos, no se entenderá comprendida entre las funciones de dirección la mera pertenencia al Consejo de Administración de la Sociedad.

c) En cuanto al donatario, deberá mantener lo adquirido y tener derecho a la exención en el Impuesto sobre el Patrimonio durante los diez años siguientes a la fecha de la escritura de donación, salvo que falleciera dentro de ese plazo".

Asimismo, tanto en la transmisión "intervivos" como "mortis causa" no se pueden efectuar disposiciones que directa o indirectamente disminuyan el valor de de la adquisición. En todo caso, de efectuarse la transmisión lucrativa habría que interpretarla de acuerdo a lo establecido en la Resolución 2/1999 de 23 de Marzo de la Dirección General de Tributos relativa a la aplicación de las reducciones en la base imponible del Impuesto de Sucesiones y Donaciones en materia de vivienda y empresa familiar.

Tercero. Con la configuración del órgano de administración en el artículo 447 del Texto Refundido de la Ley de Sociedades de Capital en el que prevalece la figura del administrador como órgano unipersonal y se excluye la posibilidad de que exista un consejo de administración, se puede cumplir una de las exigencias que establece el artículo 4.8 de la Ley del Impuesto de Patrimonio para disfrutar de la exención prevista en dicha Ley, cual es, la efectividad de la función de dirección en la SLNE. Dicha exigencia se concreta según el Real Decreto 1704/1999 en su artículo 3 al exigir que la actividad se ejerza "de forma habitual, personal y directa" (artículo 21.1.a. del Texto Refundido) y en el artículo 4.2 d) precisando "que se considerarán funciones de dirección, que deberán acreditarse fehacientemente mediante el correspondiente contrato o nombramiento, los cargo de: Presidente, Director General, Gerente, Administrador, Directores de Departamento, Consejeros y miembros del Consejo de Administración u órgano equivalente, siempre que el desempeño de cualquiera de estos cargos implique una efectiva intervención en las decisiones de la empresa".

Como es sabido el cumplimiento de este requisito de la exención no obsta a que todos los miembros del grupo familiar disfruten de la exención porque, como aclara el artículo 4.8, dos, tercer párrafo de la Ley del Impuesto sobre el Patrimonio y añade también el Real Decreto 1704/1999 ya citado en el último párrafo del artículo 4.2. d):

"Cuando la participación de la entidad sea conjunta con alguna o algunas de las personas a las que se refiere el párrafo c) de este apartado, las funciones de dirección y las remuneraciones derivadas de la misma deberán cumplirse al menos en una de las personas del grupo de parentesco, sin perjuicio de que todas ellas tengan derecho a la exención”.

Cuarto. En relación con lo anterior, se inscribe asimismo la regulación, potestativa y flexible, de la retribución del administrador en el artículo 448.2 de la Ley de Sociedades de Capital que venimos citando. Se puede cumplir así también el requisito de la función directiva efectiva. La exigencia se concreta en que el Administrador perciba por ello "una remuneración que represente más del $50 \%$ de la totalidad de los rendimientos empresariales, profesionales y de trabajo personal. A efectos del cálculo anterior, no se computarán entre los rendimientos empresariales, profesionales y del trabajo personal los rendimientos de la actividad empresarial a la que se refiere el número 1 de este apartado" 
es decir, como precisa el artículo 5.1 d) del Real Decreto 1704/1999: "no se computarán los rendimientos de actividades económicas cuyos bienes y derechos afectos disfruten de exención en este impuesto". En consecuencia, la Junta General puede ajustar la retribución del administrador a las características fiscales del mismo y cumplir la Ley de Sociedades de Capital y la Ley del Impuesto sobre el Patrimonio.

Quinto. En lo que se refiere a la configuración del objeto social regulado en el artículo 436 del Texto Refundido de la Ley de Sociedades de Capital, ya hemos adelantado que el arrendamiento o la compraventa de bienes inmuebles pueden constituir el objeto de una sociedad familiar que adopte la forma de una SLNE. Para precisar si existe actividad económica o si se produce la afectación hay que estar a lo dispuesto por el artículo 4, ocho, dos de la Ley del Impuesto sobre el Patrimonio que se remite por su parte a la Ley 35/2006, reguladora del IRPF que, en su artículo 27.2, que establece:

"A efectos de lo dispuesto en el apartado anterior, se entenderá que el arrendamiento o compraventa de inmuebles se realiza como actividad económica, únicamente cuando concurran las siguientes circunstancias:

a) Que en desarrollo de la actividad se cuente, al menos, con un local exclusivamente destinado a llevar a cabo la gestión de la actividad.

b) Que para la ordenación de aquella se utilice, al menos, una persona empleada con contrato laboral y a jornada completa".

Piénsese que, al respecto, nada impide, en la legislación laboral, que la persona empleada sea un miembro del núcleo familiar, es decir, "cónyuge, ascendientes, descendientes o colaterales de segundo grado, ya tenga su origen el parentesco en la consanguinidad, en la afinidad o en la adopción" y que la disponibilidad de un local, dado que no exige sea en propiedad, es perfectamente asumible en una sociedad de estas características.

Igualmente en cuanto al objeto social y relacionado con el aspecto anterior, se debe cumplir el requisito de que la entidad no tenga por actividad principal la gestión de un patrimonio mobiliario o inmobiliario. Esta es la exigencia más importante a cumplir puesto que de no hacerlo perdería los beneficios fiscales que ya hemos mencionado en el Impuesto de Sucesiones y Donaciones. Observemos su regulación:

El artículo 4, ocho, apartado dos, de la Ley del Impuesto de Patrimonio entiende que una entidad gestiona un patrimonio mobiliario o inmobiliario y que, por lo tanto, no realiza una actividad económica cuando concurran durante más de 90 días del ejercicio social cualquiera de las condiciones siguientes: que más de la mitad de su activo esté constituido por valores o que más de la mitad de su activo no esté afecto a actividades económicas. Así el mencionado artículo 4.8, apartado dos permite tener hasta el 50\% no afecto o constituido por valores (la Ley dice "más de la mitad ") no obstante esto supone que sólo el $50 \%$ del valor de las participaciones está exento dado que el referido artículo 4.8 dos de la Ley del Impuesto de Patrimonio, inciso final afirma:

"La exención sólo alcanzará al valor de las participaciones, determinado conforme a las reglas que se establecen en el artículo 16.uno de esta Ley, en la parte que corresponda a la proporción existente entre los activos necesarios para el ejercicio de la actividad 
empresarial o profesional, minorados en el importe de las deudas derivadas de la misma, y el valor del patrimonio neto de la entidad...”.

Para precisar si existe actividad económica o si se produce la afectación hay que estar en a lo dispuesto también por el artículo 4, ocho, dos que se remite por su parte a la Ley del IRPF en su artículo 27.2 que ya hemos trascrito anteriormente.

Es importante destacar, que el artículo 4, ocho, dos de la Ley del Impuesto del Patrimonio precisa tanto los supuestos en que no hay afectación y por tanto se pierde la exención como aquellos supuestos especiales en que se considera que sí hay afectación, como, por ejemplo, los bienes poseídos para dar cumplimiento a obligaciones legales y reglamentarias, $u$ otros elementos del activo que no se consideran afectos como los que incorporan derechos de crédito, los poseídos por sociedades de valores o los que otorguen el $5 \%$ de derechos de voto en sociedades participadas.

En lo que se refiere al futuro desarrollo de la Sociedad, ésta puede transformar su personalidad jurídica modificando los Estatutos mediante la oportuna escritura pública, por ejemplo convirtiéndose en Sociedad Limitada, según lo dispuesto en el artículo 454 de la Ley de Sociedades de Capital para lo cual requerirá simplemente un acuerdo por mayoría ordinaria de la junta general y adaptar los estatutos a lo exigida para una Sociedad de dicha naturaleza.

Sexto. El Real Decreto Ley 13/2010, de 3 de diciembre, de actuaciones en el ámbito fiscal, laboral y liberalizadoras para el fomento de la inversión y la creación de empleo, incorpora una serie de medidas que, por su trascendencia práctica, merecen ser destacadas a efectos de la constitución de sociedades en general y en concreto su artículo 5 establece medidas para agilizar y simplificar la constitución de sociedades mercantiles de capital, en particular en lo que se refiere a la constitución por medios telemáticos de sociedades de responsabilidad limitada que son, en un todo, aplicables a la SLNE y que permiten en un tiempo récord, inscribir la Sociedad en el registro Mercantil.

En este sentido, se ha reducido el coste fiscal de la Constitución de la Sociedad SLNE, y del resto de sociedades, al haber quedado exenta del Impuesto de Transmisiones Patrimoniales y Actos Jurídicos Documentados, en su modalidad de Operaciones Societarias, en virtud del artículo 3 del Real Decreto-Ley 13/2010, de 3 de diciembre que modifica su Texto Refundido, aprobado por el Real Decreto Legislativo 1/1993, de 24 de septiembre.

Al respecto se establece lo siguiente:

"Se modifica el artículo 45.I.B).11 del texto refundido de la Ley del Impuesto sobre Transmisiones Patrimoniales y Actos Jurídicos Documentados, aprobado por el Real Decreto Legislativo 1/1993, de 24 de septiembre, que queda redactado de la siguiente forma:

«La constitución de sociedades, el aumento de capital, las aportaciones que efectúen los socios que no supongan aumento de capital y el traslado a España de la sede de 
dirección efectiva o del domicilio social de una sociedad cuando ni una ni otro estuviesen previamente situados en un Estado miembro de la Unión Europea»”.

En consecuencia ninguna sociedad de capital y en particular las SLNE deben pagar el impuesto de operaciones societarias por su constitución.

Igualmente el artículo 4 del Decreto-Ley 13/2010, modifica la Ley 3/1993, de 22 de marzo, Básica de las Cámaras Oficiales de Comercio, Industria y Navegación suprimiendo el carácter obligatorio de la afiliación a las Cámaras de Comercio, Industria y Navegación y con ello se produce la desaparición del recurso cameral permanente como tributo, pasando a ser una cuota voluntaria que pagan únicamente los comerciantes, industriales o nautas que de forma voluntaria se afilien a dichas Instituciones. Así se establece en la nueva redacción del artículo 13 de la Ley 3/1993, de Cámaras:

"Artículo 13. Obligación de pago y devengo de la cuota cameral.

Estarán obligados al pago de la cuota cameral quienes ejerzan las actividades del comercio, la industria o la navegación a que se refiere el artículo 6 y decidan libremente pertenecer a una Cámara Oficial de Comercio, Industria y Navegación”.

Por lo tanto, tampoco las sociedades de capital incluidas las SLNE deberán pagar el llamado Recurso Cameral Permanente.

Séptimo. La Disposición Adicional Segunda del Texto Refundido de la Ley de Sociedades de Capital, establece la tributación de la transmisión de participaciones sociales. La cuestión tiene su relevancia porque el artículo 444 de esta Ley al establecer los requisitos subjetivos en la transmisión de las participaciones sociales prevé la posibilidad de sucesivas transmisiones. Así, prevé que se puede alcanzar el número de cinco socios como consecuencia de dichas transmisiones (artículo 444.1) y que la transmisión voluntaria "inter vivos" sólo puede hacerse a favor de personas físicas (artículo 444.2). Pero el aspecto más importante, es lo dispuesto en el artículo 444.3 cuando se afirma: "Si fueran adquiridas participaciones sociales por personas jurídicas, deberán se enajenadas a favor de personas físicas en el plazo de tres meses, contados desde la adquisición". Si no se hace así se produce "ope legis" la transformación en Sociedad Limitada sin perjuicio de la responsabilidad de los administradores al no haber adaptado los Estatutos Sociales.

Se trata, como señalamos más arriba, de una de las manifestaciones del carácter personalísimo de la SLNE, que sólo puede estar constituida por personas físicas. Por otra parte, es lógica esta exigencia, porque no tiene sentido que en una estructura social de estas características se integre una persona jurídica. En el caso de que la SLNE sea el vehículo jurídico de una sociedad familiar, aún tiene menos sentido que se integre en la misma una persona jurídica, desnaturalizándose así el modelo. Por otra parte, el artículo 444.3 de la Ley de Sociedades de Capital, quizá sin pretenderlo, permite "aparcar" durante tres meses participaciones sociales de una SLNE en una sociedad cuya recuperación se puede pactar mediante una cláusula de "retroventa" con la finalidad que se apetezca por parte de los titulares de la SLNE. Pensemos, por ejemplo, en el caso de que se intente un embargo $u$ otras medidas cautelares por deuda pendientes sobre las 
participaciones que, en el momento de la ejecución se encuentren "depositadas" en la persona jurídica. A nuestro juicio, esta posibilidad no se debía haber incluido en la Ley, debiéndose haber establecido, lisa y llanamente la prohibición de transmitir las participaciones a personas jurídicas.

En cuanto al régimen fiscal en concreto, la adicional citada se limita a remitirse al establecido para la transmisión de valores en el artículo 108 de la Ley 24/1988, de 28 de julio, del Mercado de Valores. Esto significa, en síntesis, que la transmisión está exenta del Impuesto de Transmisiones Patrimoniales y del IVA siempre que esta transmisión no suponga la transmisión encubierta de bienes inmuebles, circunstancia que se considera producida cuando de forma directa o indirecta el activo de la Sociedad esté constituido en más de un $50 \%$ por bienes inmuebles o de derechos que permitan el control de dicho activo. Al respecto señala el artículo 108.2.a):

"Cuando los valores o participaciones transmitidos o adquiridos representen partes alícuotas del capital social o patrimonio de sociedades, fondos, asociaciones y otras entidades cuyo activo esté constituido al menos en un $50 \%$ por inmuebles situados en territorio español, o en cuyo activo se incluyan valores que le permitan ejercer el control en otra entidad cuyo activo esté integrado al menos en un $50 \%$ por inmuebles radicados en España, siempre que, como resultado de dicha transmisión o adquisición, el adquirente obtenga una posición tal que le permita ejercer el control sobre esas entidades o, una vez obtenido dicho control, aumente la cuota de participación en ellas".

En estos casos, pues, se producirá la tributación efectiva por el Impuesto de Transmisiones Patrimoniales o por el IVA.

Octavo. La simplificación en el cumplimiento de los requisitos de constitución de la SLNE se amplía, en lo que se refiere al ámbito tributario, en la Disposición Adicional Tercera del Texto Refundido de la Ley de Sociedades de Capital. En concreto destacaremos los siguientes aspectos:

La creación de un Documento Único Electrónico que vehicula la información que van a exigir las administraciones públicas. Así lo establece el primer apartado de la adicional que analizamos:

“El Documento Único Electrónico (DUE) es aquel en el que se incluyen todos los datos referentes a la sociedad nueva empresa que, de acuerdo con la legislación aplicable, deben remitirse a los registros jurídicos y las Administraciones públicas competentes para la constitución de la sociedad y para el cumplimiento de las obligaciones en materia tributaria y de Seguridad Social inherentes al inicio de su actividad”.

Uno de los aspectos novedosos en la configuración de la SLNE es la posibilidad prevista de que sean los socios fundadores los que se encarguen personalmente de la tramitación del DUE ya mencionado lo que puede suponer otro ahorro en los costes de constitución. Al efecto dispone el apartado tercero de la presente adicional: 
"De conformidad con lo dispuesto en el párrafo segundo del apartado primero del artículo 440, los socios fundadores de la sociedad nueva empresa podrán manifestar al notario, previamente al otorgamiento de la escritura de constitución, su interés en realizar por sí mismos los trámites y la comunicación de los datos incluidos en el DUE o designar un representante para que lo lleve a efecto, en cuyo caso no será de aplicación lo establecido en la presente disposición adicional".

Igualmente hay que subrayar la regulación de los llamados PAIT, es decir los "puntos de asesoramiento e inicio de tramitación" que pueden contribuir mucho al objetivo de simplificar y flexibilizar la constitución de la SLNE y que se regulan en el apartado 5 de esta adicional:

"La Administración General del Estado, a través del Ministerio de Economía y Hacienda, podrá celebrar convenios de establecimiento de puntos de asesoramiento e inicio de tramitación (PAIT) de las sociedades nueva empresa con otras Administraciones públicas y entidades públicas o privadas. Los puntos de asesoramiento e inicio de tramitación serán oficinas desde las que se podrá solicitar la reserva de denominación social a que se refiere el apartado segundo del artículo 440 y se asesorará y prestarán servicios a los emprendedores, tanto en la definición y tramitación administrativa de sus iniciativas empresariales como durante los primeros años de actividad de las mismas, y en ellos se deberá iniciar la tramitación del DUE. En los convenios se establecerán los servicios de información, asesoramiento y tramitación que deben prestarse de forma gratuita y los de carácter complementario que pueden ofrecerse mediante contraprestación económica”.

Al cumplimiento del mismo objetivo se dirige la utilización de las ventanillas únicas empresariales ya existentes para la constitución de la SLNE, prevista también en el apartado 5 de la adicional:

"Los centros de ventanilla única empresariales creados al amparo del Protocolo de 26 de abril de 1999 mediante los correspondientes instrumentos jurídicos de cooperación con Comunidades Autónomas y Entidades Locales podrán realizar las funciones de orientación, tramitación y asesoramiento previstas en la presente ley para la creación y desarrollo de sociedades Nueva Empresa”.

Noveno. En lo que concierne a la fiscalidad, la Disposición Adicional Cuarta del Texto Refundido de la Ley de Sociedades de Capital, articula la colaboración social mediante la suscripción de convenios con los profesionales y los Colegios que están involucrados directamente en la constitución de sociedades, como afirma su apartado primero:

"Las Administraciones tributarias podrán hacer efectiva la colaboración social prevista en el artículo 92 de la Ley 58/2003, de 17 de diciembre, General Tributaria, así como en otras normas que la desarrollen, en la presentación de declaraciones, comunicaciones u otros documentos tributarios relacionados con la constitución e inicio de la actividad de la sociedad Nueva Empresa, a través de convenios celebrados con el Consejo General del Notariado, el Colegio de Registradores de la Propiedad, 
de Bienes Muebles y Mercantiles de España y otros colegios profesionales, así como las cámaras de comercio y los puntos de asesoramiento e inicio de tramitación (PAIT)".

Décimo. La Disposición Adicional Sexta del Texto Refundido de la Ley de Sociedades de Capital prevé todo un régimen de aplazamientos y fraccionamientos de carácter privilegiado en los impuestos que afectan a la actividad empresarial de las SLNE.

Al respecto, la operatividad del primer apartado de esta adicional, ha quedado sin eficacia desde el momento en que, como hemos indicado más arriba, el artículo 3 del Real Decreto-Ley 13/2010, de 3 de diciembre, ha declarado exenta la constitución de sociedades como hecho imponible del Impuesto de Transmisiones Patrimoniales en su modalidad de operaciones societarias.

Sin embargo, el resto de medidas son incentivos fiscales muy importantes a favor de que las sociedades familiares se configuren como SLNE. Así hay que poner de relieve la novedad del privilegio que se concede a estas sociedades de obtener mediante solicitud el aplazamiento de las dos primeras liquidaciones del impuesto de Sociedades que deberán ingresarse "a los 12 y seis meses, respectivamente, desde la finalización de los plazos para presentar la declaración-liquidación correspondiente a cada uno de dichos períodos" según lo que dispone el apartado primero de la adicional.

En este mismo apartado se prevé, igualmente previa solicitud y sin garantías el aplazamiento y fraccionamiento: "de los ingresos por de retenciones o ingresos a cuenta del Impuesto sobre la Renta de las Personas Físicas que se devenguen en el primer año desde su constitución".

Por último, también se concede en el apartado de esta adicional a las SLNE el privilegio de no ingresar los pagos fraccionados del Impuesto de Sociedades a los que se refiere el artículo 45 del Texto Refundido que regula dicho impuesto correspondientes a los dos primeros periodos impositivos, pudiendo ingresar, por tanto, directamente la deuda tributaria sin adelantar dichos pagos.

\section{Conclusiones}

Visto el anterior marco normativo, creemos que para la promoción de la sociedad familiar en el ámbito de la pequeña y mediana empresa deben tenerse en cuenta las siguientes consideraciones y sería necesario, desde una perspectiva de "lege ferenda", adoptar las siguientes medidas:

1.- Valoración general. El cuadro general de incentivos fiscales, facilidades y simplicidad de requisitos que hemos descrito no tiene parangón con ningún otro régimen societario. Por lo tanto es altamente recomendable que las sociedades familiares que estén en los límites que fija la Ley de Sociedades de Capital se constituyan como SLNE. 
2.- Por lo que acabamos de señalar creemos que resulta especialmente lesivo para dicho objetivo, el límite máximo de capital establecido para la SLNE y fijado en $120.202 €$. Hoy en día esta cantidad resulta excesivamente pequeña y limita en exceso el ámbito empresarial que puede beneficiarse de las ventajas establecidas para dicha sociedad.

3.- Debe suprimirse la exigencia de que todo el capital social esté desembolsado en aportaciones dinerarias. Esto impide que sea posible aportar activos que ya existan y que, sin embargo, lo cual puede facilitar mucho el inicio de la actividad, como por ejemplo, un local, un nombre comercial o determinada maquinaria. No se entiende bien porqué se establece dicha exigencia que, sin embargo, no deben cumplir otras fórmulas societarias.

4.- La SLNE ya tiene suficientes beneficios fiscales y facilidades en el momento de su nacimiento, ahora lo que es necesario es que los mantenga en el momento de su desarrollo. Es decir, una vez iniciada la actividad donde se deben concentrar las ayudas es en los primeros años de crecimiento. Por eso consideramos que se debe estudiar un tipo superreducido del Impuesto de Sociedades para esta clase de entidades con un límite temporal, por ejemplo, los primeros cinco periodos impositivos. El porcentaje actualmente aplicable para las empresas de reducida dimensión, 20\% (para las bases imponibles entre 0 y 120.202,4 €), siempre que mantengan el empleo y cumplan los demás requisitos establecidos en el Capítulo XII, del Título VII del Texto Refundido de la Ley del Impuesto de Sociedades, es demasiado elevado aún.

5.- Lo mismo podemos decir, respecto de las obligaciones formales. Durante el mismo lapso de tiempo (cinco años) deberían simplificarse al máximo, las obligaciones a las que se refiere en general, el artículo 29 de la Ley General Tributaria, Ley 58/2003 y su desarrollo en los artículos 2 a 58 del Reglamento de Gestión e Inspección aprobado por el Real Decreto 1065/2007, de 27 de julio, además de las que se prevén en cada uno de los impuestos en particular. Como línea de actuación debería seguirse la establecida con la implantación de los puntos de asesoramiento e inicio de tramitación (PAIT) prevista en la Disposición Adicional Quinta de la Ley de Sociedades de Capital para que tuviera carácter permanente para las entidades constituidas como SLNE. 